\title{
Differential cross section for the Higgs boson production in 4-lepton channel and $k_{T}$-factorization
}

\author{
Vaibhav S. Rawoot* \\ The Institute of Mathematical Sciences, \\ IV Cross Road, CIT Campus, Chennai 600 113, India. \\ E-mail: vaibhavrawoot@gmail.com
}

\section{Rashidul Islam}

The Institute of Mathematical Sciences,

IV Cross Road, CIT Campus, Chennai 600 113, India.

E-mail: rislameitt.ernet.in

\section{Mukesh Kumar}

National Institute for Theoretical Physics,

School of Physics and Mandelstam Institute for Theoretical Physics,

University of the Witwatersrand, Johannesburg, Wits 2050, South Africa.

E-mail: mukesh.kumar@cern.ch

\begin{abstract}
We present our study of the differential cross section for the Higgs boson production in $k_{T^{-}}$ factorization framework. The $k_{T}$-factorization formalism includes a convolution over unintegrated parton distribution functions (uPDF) and off-shell parton level matrix element. The offshell matrix element calculated considering initial gluons to be off-shell. We have considered only gluon fusion process which is dominant production mechanism for the Higgs boson production at LHC. We have used Ciafaloni-Catani-Fiorani-Marchesini (CCFM) uPDF based on CCFM evolution equations. We have compared our results with fixed order estimates up to NNLO+NNLL obtained using HRes tool within collinear factorization framework as well as with the ATLAS and CMS measurements of the corresponding differential distributions. This study will play an important role in understanding differential cross section within $k_{T}$-factorization framework.
\end{abstract}

QCD Evolution 2016

May 30-June 03, 2016

National Institute for Subatomic Physics (Nikhef), Amsterdam

\footnotetext{
* Speaker.
} 


\section{Introduction}

The discovery of a $125 \mathrm{GeV}$ candidate of the Standard Model (SM) Higgs boson by the ATLAS and CMS experiments $[1,2]$ opened a new plethora of studies. It has been established beyond doubt that the new particle is a spin-0 Higgs boson rather than a spin-2 particle [3]. In the minimal SM, the electroweak symmetry breaking is achieved via the Higgs mechanism through a single complex scalar doublet which leads to only one neutral physical scalar, $h$. Non-minimal models assume the existence of additional charged and neutral scalar Higgs particles. Hence on one hand we are concerned about its couplings to fermions and gauge bosons and the results seems to confirm consistency with the expected SM values so far [2]. On the other hand it opens the possibility to probe the production channel of the said Higgs boson. Here we are concerned with the latter aspect of the Higgs boson studies.

At the LHC conditions, the gluon-gluon fusion channel, $g g \rightarrow h$ is the most dominant one for the inclusive Higgs boson production [4]. Clearly, the gluon-gluon fusion to the Higgs production is strongly dependent on the gluon density $x f_{g}\left(x, \mu_{F}^{2}\right)$ in a proton which are usually described as a function of the Bjorken variable, $x$ and hard scale, $\mu_{F}^{2}$ within the framework of the DGLAP evolution equation [5]. The cross sections are calculated from the hard matrix elements convoluted with gluon density functions. This is the so called collinear factorization approach. However, at the LHC energies, it is more appropriate to use the parton densities which are explicit functions of it's transverse momentum, $k_{T}$ [6] (the so called unintegrated, i.e., $k_{T}$ dependent parton distribution functions or uPDFs in short). These parton densities are described by the BFKL evolution equation [7] for very small $x$ or the CCFM evolution equation [8] which is valid for both small and large $x^{1}$. As in the case of the collinear factorization, it is also possible to factorize the cross section into a convolution of the hard matrix elements and gluon uPDFs. However, in this case the matrix elements have to be taken off-shell and the convolution should also be over $k_{T}$. This generalized factorization is called $k_{T}$-factorization [9].

Recently the ATLAS and CMS collaborations at the LHC presented a measurement of fiducial differential cross section of the Higgs boson in the four-lepton decay channel [10,11]. In particular, they presented a measurement of differential cross section in transverse momentum and rapidity of the Higgs boson decay into four-leptons. The measurements seems to be well in agreement with the theoretical calculation based on the collinear factorization approach with fixed order calculation up to the next to next leading order (NNLO) and including soft gluon resummation at small transverse momenta up to the next to next leading logarithm (NNLL). The $k_{T}$-factorization approach where the effect of intrinsic transverse momentum is taken into account has been a recent interest in the case of the Higgs boson production [12]. The $k_{T}$-factorization approach provides an advantage to understand an intrinsic dynamic of partons usually formulated in the form of uPDFs [13].

Here we have presented a study for the differential cross section of the Higgs boson in the four-lepton channel using $k_{T}$-factorization approach. We have used the CCFM evolution equations for the uPDFs. We have evaluated off-shell matrix element for the partonic subprocess $g^{*} g^{*} \rightarrow h \rightarrow$ $Z Z \rightarrow 4 \ell, \ell=e, \mu$. We presented our results for the differential cross section of the Higgs boson production in the four-lepton channel in the framework of $k_{T}$-factorization.

\footnotetext{
${ }^{1}$ CCFM evolution is equivalent to BFKL evolution in the limit of very small $x$, whereas similar to the DGLAP evolution for large $x$ and high $\mu_{F}^{2}$
} 


\section{Formalism}

To estimate differential cross section for the Higgs boson production in four-lepton decay channel, we have calculated off-shell matrix element for process $g^{*} g^{*} \rightarrow h \rightarrow Z Z \rightarrow 4 \ell$. We have considered effective field theory approach in our calculation. Effective Lagrangian for the gluon coupling to the Higgs boson is

$$
\mathscr{L}_{g g h}=\frac{\alpha_{S}}{12 \pi}\left(\sqrt{2} G_{F}\right)^{1 / 2} G_{\mu \nu}^{a} G^{a \mu v} h,
$$

where $\alpha_{s}$ and $G_{F}$ are the strong coupling constant and Fermi coupling constant respectively. $G_{\mu \nu}^{a}$ is the gluon field strength tensor and $h$ is the Higgs scalar field.

Triangle vertex for $\mathrm{Hg}$ effective coupling in the infinite top mass limit is

$$
T_{g g H}^{\mu v, a b}\left(k_{1}, k_{2}\right)=i \delta^{a b} \frac{\alpha_{s}}{3 \pi}\left(\sqrt{2} G_{F}\right)^{1 / 2}\left[k_{2}^{\mu} k_{1}^{v}-\left(k_{1} \cdot k_{2}\right) g^{\mu v}\right] .
$$

Using Eq. (2.2) and considering transverse momentum of initial gluon to be non-zero $k_{1}^{2}=-\mathbf{k}_{\perp 1}^{2} \neq$ 0 and $k_{2}^{2}=-\mathbf{k}_{\perp 2}^{2} \neq 0$ we derived matrix element for process $g^{*}\left(k_{1}\right) g^{*}\left(k_{2}\right) \rightarrow h \rightarrow Z Z \rightarrow \ell\left(p_{1}\right) \bar{\ell}\left(p_{2}\right)$ $\ell^{\prime}\left(p_{3}\right) \bar{\ell}^{\prime}\left(p_{4}\right)$. Matrix element we have obtained is

$$
\begin{aligned}
|\mathscr{M}|^{2}=\frac{2}{9} \frac{\alpha_{s}^{2}}{\pi^{2}} \frac{m_{Z}^{4}}{v^{4}} \frac{\left[\left(\mathbf{k}_{\perp 1}+\mathbf{k}_{\perp 2}\right)^{2}+\hat{s}\right]^{2}}{\left(\hat{s}-m_{h}^{2}\right)^{2}+\Gamma_{h}^{2} m_{h}^{2}} \cos ^{2} \phi \times \\
\frac{\left[\left(p_{1} \cdot p_{4}\right)\left(p_{2} \cdot p_{3}\right)\left\{2 g_{L}^{2} g_{R}^{2}\right\}+\left(p_{1} \cdot p_{3}\right)\left(p_{2} \cdot p_{4}\right)\left\{g_{L}^{4}+g_{R}^{4}\right\}\right]}{\left[\left(2 p_{1} \cdot p_{2}-m_{Z}^{2}\right)^{2}+\Gamma_{Z}^{2} m_{Z}^{2}\right]\left[\left(2 p_{3} \cdot p_{4}-m_{Z}^{2}\right)^{2}+\Gamma_{Z}^{2} m_{Z}^{2}\right]},
\end{aligned}
$$

and

$$
g_{L}=\frac{g_{W}}{\cos \theta_{W}}\left(-\frac{1}{2}+\sin ^{2} \theta_{W}\right), \quad g_{R}=\frac{g_{W}}{\cos \theta_{W}} \sin ^{2} \theta_{W}, \quad \text { and } \quad v=\left(\sqrt{2} G_{F}\right)^{-1 / 2},
$$

where $\Gamma_{h}$ and $\Gamma_{Z}$ are total decay width of the Higgs boson and $Z$ boson respectively. $k_{\perp 1}$ and $k_{\perp 2}$ are intrinsic transverse momentum of the initial gluons. $\phi$ is the azimuthal angle between transverse momentum of the initial gluons $\mathbf{k}_{\perp 1}$ and $\mathbf{k}_{\perp 2} . m_{Z}$ and $m_{h}$ are the Higgs boson and $\mathrm{Z}$ boson masses respectively. Partonic center of mass energy is denoted by $\hat{s}$. $\theta_{W}$ and $g_{W}$ are weak mixing angle and coupling of weak interaction respectively.

The summation over polarization of off-shell initial gluon considered according to $k_{T}$-factorization prescription is

$$
\sum \varepsilon^{v} \varepsilon^{* \mu}=\frac{\mathbf{k}_{\perp}{ }^{\mu} \mathbf{k}_{\perp}{ }^{v}}{\mathbf{k}_{\perp}^{2}} .
$$

Within the framework of $k_{T}$-factorization, cross section for the process $p\left(P_{1}\right)+p\left(P_{2}\right) \rightarrow h \rightarrow$ $Z Z \rightarrow \ell\left(p_{1}\right) \bar{\ell}\left(p_{2}\right) \ell^{\prime}\left(p_{3}\right) \bar{\ell}^{\prime}\left(p_{4}\right)$ is written as

$$
\begin{aligned}
\sigma= & \int \frac{d x_{1}}{x_{1}} \frac{d x_{2}}{x_{2}} \frac{d^{2} \mathbf{k}_{\perp 1}}{\pi} \frac{d^{2} \mathbf{k}_{\perp 2}}{\pi} \frac{1}{x_{1} x_{2} s\left(2^{12}\right)(\pi)^{8}} \frac{d^{3} p_{1}}{2 p_{10}} \frac{d^{3} p_{2}}{2 p_{20}} \frac{d^{3} p_{3}}{2 p_{30}} \frac{d^{3} p_{4}}{2 p_{40}} \\
& \times|\overline{\mathscr{M}}|^{2} \delta^{4}\left(k_{1}+k_{2}-p_{1}-p_{2}-p_{3}-p_{4}\right) f_{g}\left(x_{1}, \mathbf{k}_{\perp 1}^{2}\right) f_{g}\left(x_{2}, \mathbf{k}_{\perp 2}^{2}\right) .
\end{aligned}
$$


Simplifying Eq. (2.6) we obtained our final expression for cross section as

$$
\begin{aligned}
\sigma= & \int d y_{1} d y_{2} d y_{3} d y_{4} d \mathbf{p}_{1 T}^{2} d \mathbf{p}_{2 T}^{2} d \mathbf{p}_{3 T}^{2} d \mathbf{k}_{\perp 1}^{2} d \mathbf{k}_{\perp 2}^{2} \frac{d \phi_{1}}{2 \pi} \frac{d \phi_{2}}{2 \pi} \\
& \times \frac{1}{\left(2^{12}\right) \pi^{5}\left(x_{1} x_{2} s\right)^{2}}|\overline{\mathscr{M}}|^{2} f_{g}\left(x_{1}, \mathbf{k}_{\perp 1}^{2}\right) f_{g}\left(x_{2}, \mathbf{k}_{\perp 2}^{2}\right),
\end{aligned}
$$

with longitudinal momentum fraction $x_{1}$ and $x_{2}$ of initial gluons are

$$
\begin{gathered}
x_{1}=\frac{\left|\mathbf{p}_{1 T}\right|}{\sqrt{s}} e^{y_{1}}+\frac{\left|\mathbf{p}_{2 T}\right|}{\sqrt{s}} e^{y_{2}}+\frac{\left|\mathbf{p}_{3 T}\right|}{\sqrt{s}} e^{y_{3}}+\frac{\left|\mathbf{p}_{4 T}\right|}{\sqrt{s}} e^{y_{4}}, \\
x_{2}=\frac{\left|\mathbf{p}_{1 T}\right|}{\sqrt{s}} e^{-y_{1}}+\frac{\left|\mathbf{p}_{2 T}\right|}{\sqrt{s}} e^{-y_{2}}+\frac{\left|\mathbf{p}_{3 T}\right|}{\sqrt{s}} e^{-y_{3}}+\frac{\left|\mathbf{p}_{4 T}\right|}{\sqrt{s}} e^{-y_{4}},
\end{gathered}
$$

and

$$
\mathbf{k}_{\perp 1}+\mathbf{k}_{\perp 2}=\mathbf{p}_{1 T}+\mathbf{p}_{2 T}+\mathbf{p}_{3 T}+\mathbf{p}_{4 T},
$$

where $\phi_{1}$ and $\phi_{2}$ are the azimuthal angle of $\mathbf{k}_{\perp 1}$ and $\mathbf{k}_{\perp 2}$ respectively. $y$ and $p_{T}$ are rapidity and transverse momentum of the final state leptons. Hadronic center of mass energy is denoted by $s$.

We have calculated cross section for the Higgs boson decay as a function of rapidity of fourleptons $(y)$ and transverse momentum of the four-leptons $\left(p_{T}\right)$. We have used Eq. (2.7) together with off-shell matrix element given in Eq. (2.3) for process $g^{*} g^{*} \rightarrow h \rightarrow Z Z \rightarrow 4 \ell, \ell=e, \mu$, to calculate differential cross section.

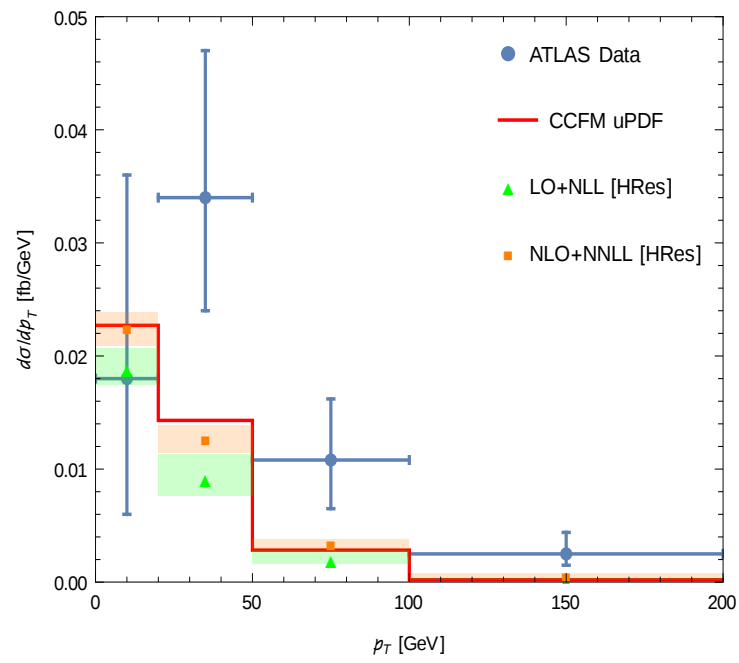

(a)

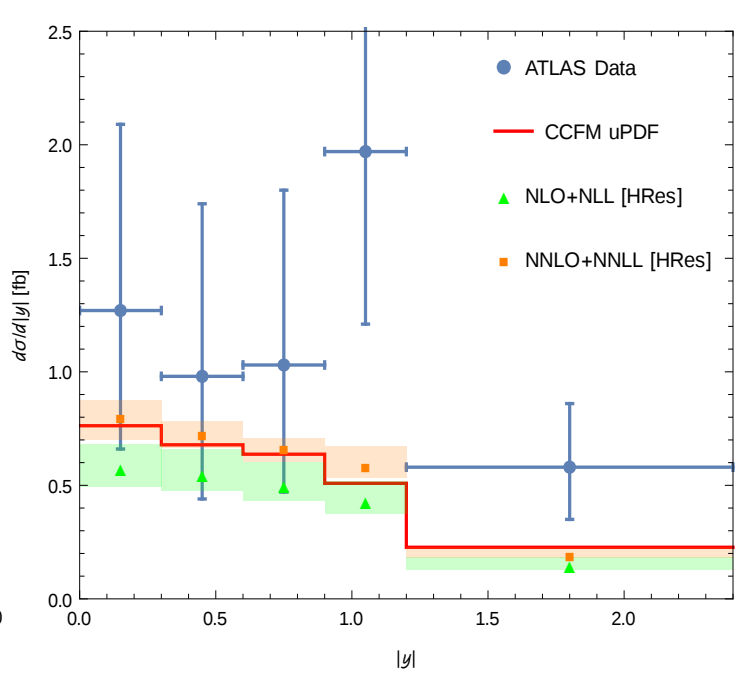

(b)

Figure 1: Differential cross section of the Higgs boson production in four-lepton decay channel at $\sqrt{s}=8$ $\mathrm{TeV}$. (a) and (b) are the differential distributions in transverse momentum of the four-leptons $\left(p_{T}\right)$ and rapidity $(y)$ of the four-leptons respectively. Solid line (red) is a results obtained using $k_{T}$-factorization approach with CCFM gluon uPDFs. Filled triangle and filled square points corresponds to estimated obtained using HRes tool up to NNLO+NNLL accuracy and shaded region corresponds to scale uncertainty in renormalization and factorization scale. Experimental data points are from ATLAS. The error bars on the data points shows total (statistical $\oplus$ systematic) uncertainty. 


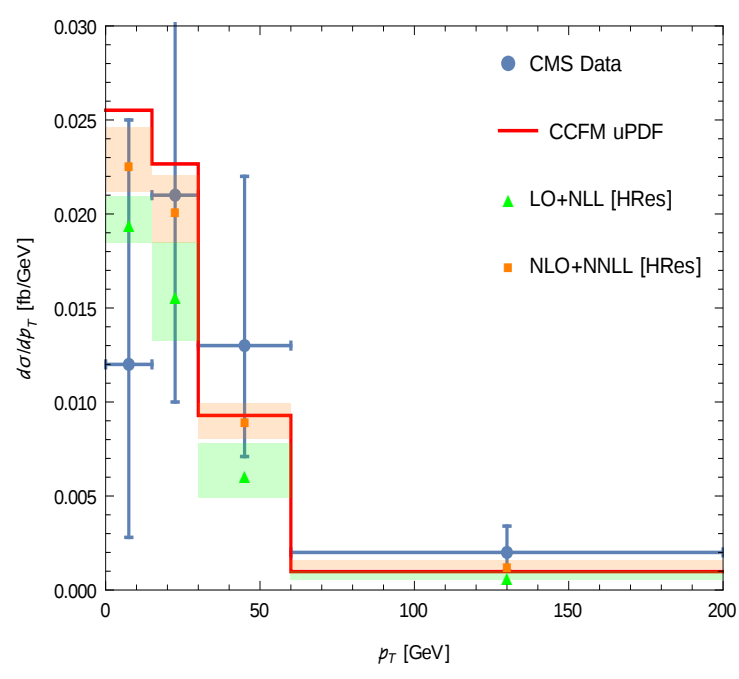

(a)

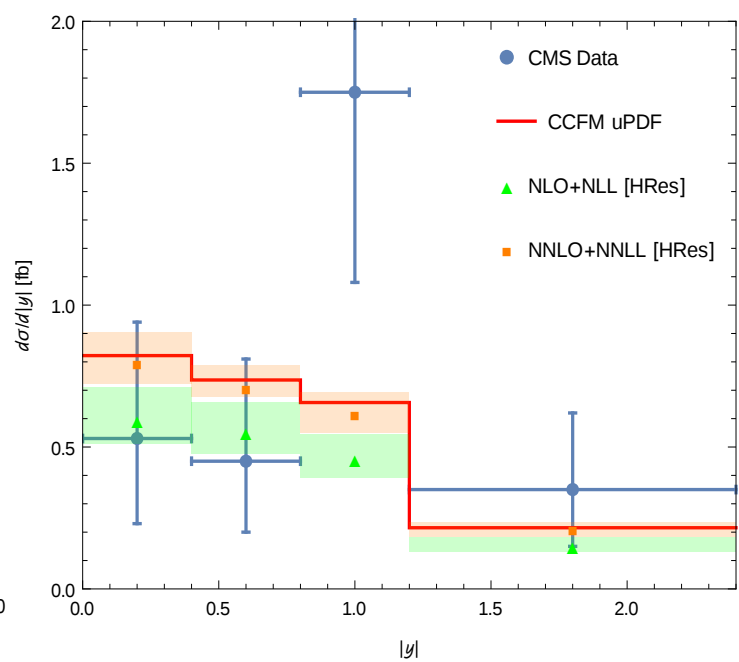

(b)

Figure 2: Differential cross section of the Higgs boson production in four-lepton decay channel at $\sqrt{s}=8$ $\mathrm{TeV}$. (a) and (b) are the plots of differential distributions in transverse momentum of the four-leptons $\left(p_{T}\right)$ and rapidity $(y)$ of the four-leptons respectively. Solid line (red) is a results obtained using $k_{T}$-factorization approach with CCFM gluon uPDFs. Filled triangle and filled square points corresponds to estimated obtained using HRes tool up to NNLO+NNLL accuracy and shaded region corresponds to scale uncertainty in renormalization and factorization scale. Experimental data points are from CMS. The error bars on the data points shows total (statistical $\oplus$ systematic) uncertainty

\section{Numerical Estimates}

We have calculated differential cross section for the Higgs boson decay into four leptons at $\sqrt{s}=8 \mathrm{TeV}$. Total decay width and mass of the Higgs boson is set to be equal to $4.0 \mathrm{MeV}$ and $125.09 \mathrm{GeV}$ respectively [14]. Our results are compared with recent experimental data from ATLAS and CMS collaborations [10,11]. We have also compared our estimates with a results obtained using HRes tool [15] which gives a fixed order cross section calculated up to next to next to leading order plus next to next leading logarithm (NNLO+NNLL) accuracy within collinear factorization formalism.

In Figure 1, we have given an estimates obtained using $k_{T}$-factorization approach to compare with the ATLAS measurements [10]. Our results obtained using $k_{T}$ factorization are close to NNLO+NNLL results obtained using HRes tool. We have done a comparison for both $p_{T}$ and $y$ differential distribution. In Figure 2, our results are compared for CMS experiment [11]. We see a similar behaviour in the comparison with the data from the CMS experiment as well. Our results are consistently close to NNLO+NNLL results which are fixed order calculation within collinear factorization approach. This can be explained considering the fact that the main part of higher order corrections included in the $k_{T}$-factorization approach [12].

\section{Conclusion}

Here we have presented our results of the differential cross section for the Higgs boson produc- 
tion within $k_{T}$-factorization approach. We have compared our results with the fixed order estimates up to the order NNLO+NNLL within collinear factorization obtained using HRes tool. We have plotted our results against recent experimental measurements of the differential cross section for the Higgs boson production in four-lepton decay channel from the ATLAS and CMS Collaborations. In summary, we have done a phenomenological study for the case of the Higgs boson production in the four-lepton decay channel considering the framework of $k_{T}$-factorization. In this phenomenological study we have used CCFM unintegrated parton densities.

\section{Acknowledgements}

Authors would like to extend sincere gratitude to V. Ravindran for continuous support and fruitful discussions during the course of this work and A. Lipatov for providing their codes of a similar study for diphoton distributions. VR would like to thank Giancarlo Ferrera for useful discussions on HRes tool and related topics. RI would also like to acknowledge the hospitality provided by the Institute of Mathematical Sciences, Chennai, India where a part of work has been done.

\section{References}

[1] G. Aad et al., Observation of a new particle in the search for the Standard Model Higgs boson with the ATLAS detector at the LHC, Phys. Lett. B716 (2012) 1-29.

S. Chatrchyan et al., Observation of a new boson at a mass of $125 \mathrm{GeV}$ with the CMS experiment at the LHC, Phys. Lett. B716 (2012) 30-61.

[2] V. Khachatryan et al., Precise determination of the mass of the Higgs boson and tests of compatibility of its couplings with the standard model predictions using proton collisions at 7 and 8 TeV, Eur. Phys. J. C75 (2015) 212.

G. Aad et al., Combined Measurement of the Higgs Boson Mass in pp Collisions at $\sqrt{\mathrm{s}}=7$ and $8 \mathrm{TeV}$ with the ATLAS and CMS Experiments, Phys. Rev. Lett. 114 (2015) 191803.

[3] S. Chatrchyan et al., Study of the Mass and Spin-Parity of the Higgs Boson Candidate Via Its Decays to Z Boson Pairs, Phys. Rev. Lett. 110 (2013) 081803.

G. Aad et al., Evidence for the spin-0 nature of the Higgs boson using ATLAS data, Phys. Lett. B726 (2013) 120-144.

V. Khachatryan et al., Constraints on the spin-parity and anomalous HVV couplings of the Higgs boson in proton collisions at 7 and 8 TeV, Phys. Rev. D92 (2015) 012004.

G. Aad et al., Study of the spin and parity of the Higgs boson in diboson decays with the ATLAS detector, Eur. Phys. J. C75 (2015) 476.

G. Aad et al., Measurements of the Higgs boson production and decay rates and coupling strengths using pp collision data at $\sqrt{s}=7$ and 8 TeV in the ATLAS experiment, Eur. Phys. J. C76 (2016) 6.

[4] F. Wilczek, Decays of Heavy Vector Mesons Into Higgs Particles, Phys. Rev. Lett. 39 (1977) 1304.

H. M. Georgi, S. L. Glashow, M. E. Machacek and D. V. Nanopoulos, Higgs Bosons from Two Gluon Annihilation in Proton Proton Collisions, Phys. Rev. Lett. 40 (1978) 692.

J. R. Ellis, M. K. Gaillard, D. V. Nanopoulos and C. T. Sachrajda, Is the Mass of the Higgs Boson About 10-GeV?, Phys. Lett. B83 (1979) 339-344. 
T. G. Rizzo, Gluon Final States in Higgs Boson Decay, Phys. Rev. D22 (1980) 178.

D. Graudenz, M. Spira and P. M. Zerwas, QCD corrections to Higgs boson production at proton proton colliders, Phys. Rev. Lett. 70 (1993) 1372-1375.

M. Spira, A. Djouadi, D. Graudenz and P. M. Zerwas, Higgs boson production at the LHC, Nucl. Phys. B453 (1995) 17-82.

[5] V. N. Gribov and L. N. Lipatov, Deep inelastic e p scattering in perturbation theory, Sov. J. Nucl. Phys. 15 (1972) 438-450.

L. N. Lipatov, The parton model and perturbation theory, Sov. J. Nucl. Phys. 20 (1975) 94-102.

G. Altarelli and G. Parisi, Asymptotic Freedom in Parton Language, Nucl. Phys. B126 (1977) 298-318.

Y. L. Dokshitzer, Calculation of the Structure Functions for Deep Inelastic Scattering and e+eAnnihilation by Perturbation Theory in Quantum Chromodynamics., Sov. Phys. JETP 46 (1977) 641-653.

[6] B. Andersson et al., Small x phenomenology: Summary and status, Eur. Phys. J. C25 (2002) 77-101.

J. R. Andersen et al., Small x phenomenology: Summary and status, Eur. Phys. J. C35 (2004) 67-98.

J. R. Andersen et al., Small x Phenomenology: Summary of the 3rd Lund Small x Workshop in 2004, Eur. Phys. J. C48 (2006) 53-105.

[7] E. A. Kuraev, L. N. Lipatov and V. S. Fadin, Multi - Reggeon Processes in the Yang-Mills Theory, Sov. Phys. JETP 44 (1976) 443-450.

E. A. Kuraev, L. N. Lipatov and V. S. Fadin, The Pomeranchuk Singularity in Nonabelian Gauge Theories, Sov. Phys. JETP 45 (1977) 199-204.

I. I. Balitsky and L. N. Lipatov, The Pomeranchuk Singularity in Quantum Chromodynamics, Sov. J. Nucl. Phys. 28 (1978) 822-829.

[8] M. Ciafaloni, Coherence Effects in Initial Jets at Small q**2 /s, Nucl. Phys. B296 (1988) 49-74.

S. Catani, F. Fiorani and G. Marchesini, QCD Coherence in Initial State Radiation, Phys. Lett. B234 (1990) 339-345.

S. Catani, F. Fiorani and G. Marchesini, Small x Behavior of Initial State Radiation in Perturbative QCD, Nucl. Phys. B336 (1990) 18-85.

G. Marchesini, QCD coherence in the structure function and associated distributions at small x, Nucl. Phys. B445 (1995) 49-80.

[9] L. V. Gribov, E. M. Levin and M. G. Ryskin, Semihard Processes in QCD, Phys. Rept. 100 (1983) $1-150$.

E. M. Levin, M. G. Ryskin, Yu. M. Shabelski and A. G. Shuvaev, Heavy quark production in semihard nucleon interactions, Sov. J. Nucl. Phys. 53 (1991) 657.

S. Catani, M. Ciafaloni and F. Hautmann, High-energy factorization and small $x$ heavy flavor production, Nucl. Phys. B366 (1991) 135-188.

J. C. Collins and R. K. Ellis, Heavy quark production in very high-energy hadron collisions, Nucl. Phys. B360 (1991) 3-30.

[10] G. Aad et al., Fiducial and differential cross sections of Higgs boson production measured in the four-lepton decay channel in pp collisions at $\sqrt{s}=8 \mathrm{TeV}$ with the ATLAS detector, Phys. Lett. B738 (2014) 234-253. 
[11] V. Khachatryan et al., Measurement of differential and integrated fiducial cross sections for Higgs boson production in the four-lepton decay channel in pp collisions at $\sqrt{s}=7$ and $8 \mathrm{TeV}, \mathrm{JHEP} 04$ (2016) 005.

[12] A. V. Lipatov and N. P. Zotov, Higgs boson production at hadron colliders in the $k(T)$-factorization approach, Eur. Phys. J. C44 (2005) 559-566.

M. G. Ryskin, A. G. Shuvaev and Yu. M. Shabelski, Charm hadroproduction in $k(T)$ factorization approach, Phys. Atom. Nucl. 64 (2001) 120-131.

[13] A. V. Lipatov, M. A. Malyshev and N. P. Zotov, Phenomenology of $k_{t}$-factorization for inclusive Higgs boson production at LHC, Phys. Lett. B735 (2014) 79-83.

[14] K. A. Olive et al., Review of Particle Physics, Chin. Phys. C38 (2014) 090001.

[15] D. de Florian, G. Ferrera, M. Grazzini and D. Tommasini, Higgs boson production at the LHC: transverse momentum resummation effects in the $\mathrm{H}->2$ gamma, $\mathrm{H}->\mathrm{WW}->\ln \mathrm{ln} \ln$ and $\mathrm{H}->\mathrm{ZZ}->4 \mathrm{l}$ decay modes, JHEP 06 (2012) 132.

M. Grazzini and H. Sargsyan, Heavy-quark mass effects in Higgs boson production at the LHC, JHEP 09 (2013) 129. 